\title{
A 10-year analysis of metastatic prostate cancer as an initial presentation in an underserved population
}

\author{
Andrew G. Winer, John P. Sfakianos, Llewellyn M. Hyacinthe, Brian K. McNeil \\ Department of Urology, SUNY Downstate Medical Center and Kings County Medical Center, Brooklyn, \\ NY, USA
}

\section{ABSTRACT}

Objective: To analyze patients from an underserved area who presented initially with metastatic prostate cancer in order to identify patients in our population who would suffer greatly if PSA screening was eliminated.

Materials and Methods: A prospectively maintained androgen deprivation therapy database from an inner city municipal hospital was queried to identify patients who presented with metastatic prostate cancer. We identified 129 individuals from 1999 to 2009 eligible for study. Those who underwent previous treatment for prostate cancer were excluded. We examined metastatic distribution and analyzed survival using Kaplan Meier probability curves.

Results: The median age of presentation was 68 with a median Gleason sum of 8 per prostate biopsy. Thirty-two patients presented with hydronephrosis with a median creatinine of 1.79, two of whom required emergent dialysis. Of those patients who underwent radiographic imaging at presentation, 35.5\% (33/93) had lymphadenopathy suspicious for metastasis, 16.1\% (15/93) had masses suspicious for visceral metastases. Of the patients who underwent a bone scan 93\% (118/127) had positive findings with 7.9\% (10/127) exhibiting signs of cord compression. The 2 and 5- year cancer specific survival was $92.1 \%$ and $65.6 \%$, respectively.

Conclusions: In this study we have highlighted a group of men in an underserved community who presented with aggressive and morbid PCa despite widespread acceptance of PSA screening.

\section{ARTICLE INFO}

Key words:

Prostatic Neoplasms;

Prostate-Specific Antigen;

Mass Screening; Medically

Underserved Area

Int Braz J Urol. 2014; 40: 316-21

Submitted for publication:

August 13, 2013

Accepted after revision:

March 22, 2014

\section{INTRODUCTION}

Prostate cancer $(\mathrm{PCa})$ remains among the most common causes of cancer related deaths in North American men with an estimated 28,170 deaths in 2012 (1). Since the inception of PSA screening in the early 1980's, a stage and grade migration towards diagnosing lower risk PCa has been identified. With this stage migration it was observed a lower incidence of metastatic disease and improved mortality rates (2). Etzioni et al. de- monstrated a decrease in metastatic disease from 77 per 100,000 patients in 1990 to 37 per 100,000 patients in 2000 (3).

Despite an overall trend towards organ confined, lower risk disease, the incidence and mortality rates of PCa in men of lower socioeconomic status (SES) and African American race has remained disproportionately high and relatively unchanged throughout the PSA screening era. A retrospective study looking at low income, uninsured men in California suggested that despite 
widespread use of PSA screening, clinical $\mathrm{T}$ stage, Gleason scores, and rates of metastatic disease remained unchanged over time (4). Another study from Brazil investigated the impact of screening for PCa versus traditional referral for cancer treatment on clinical and pathological features, and found that cancers detected through screening proved to be significantly more favorable (5).

Race also appears to contribute to the disproportionate rates of incidence and mortality of PCa. Jemal et al. demonstrated that over a 5-year span African American men were 1.5 times more likely to be diagnosed with PCa and 2.4 times more likely to die from the disease than white men (6).

Considering recent controversies regarding PSA screening, we sought to identify patients in an underserved population who presented initially with metastatic prostate cancer (mPCa) to identify who would suffer if PSA screening was eliminated.

\section{MATERIAL AND METHODS}

After obtaining Institutional Review Board approval we queried a prospectively maintained androgen deprivation therapy database from an inner city municipal hospital to identify patients who at initial presentation were found to have a diagnosis of mPCa. All patients in our study had a prostate biopsy confirming the presence of cancer. $\mathrm{mPCa}$ was confirmed with either a bone scan that was positive for disease or a CT scan with evidence of visceral metastasis. Imaging was also used to evaluate lymph node status. A board certified Radiologist reviewed all images.

We identified 202 individuals from 1999 to 2009 with $\mathrm{mPCa}$. Patients who had imaging studies performed within 30 days of PCa diagnosis were included in our study. Individuals who were previously treated for PCa were excluded. One hundred and twenty nine patients met our inclusion criteria and were included in our study.

We examined median age at diagnosis, Gleason score, PSA, creatinine level, hematocrit and the need for hemodialysis at the time of presentation. We examined the metastatic distribution in our patient population using nuclear bone imaging and CT scans. Unfortunately, the reporting system at our hospital does not give details such as percentage of $\mathrm{PCa}$ within fragment; we simply get a Gleason score. Also, many of the biopsies performed for men with mPCa upon presentation included only one or two cores in order to get a tissue diagnosis prior to starting systemic therapy. Therefore, all we have to report is the Gleason score, which is mentioned in the results section below.

All patients were treated with androgen deprivation initially with an androgen receptor blocker or ketoconazole for several weeks, which was given at the discretion of the treating physician. Following initial treatment, all patients were placed on an LHRH agonist. Biochemical failure was defined as a rise in serum PSA from nadir levels with a castrate testosterone level less than $50 \mathrm{ng} / \mathrm{mL}$. Individuals with evidence of biochemical failure were referred to medical oncology for initiation of secondary treatment. Secondary treatment was administered at the discretion of the treating medical oncologist.

Using SPSS 17.0 we constructed Kaplan Meier probability curves for overall survival and cancer specific survival.

\section{RESULTS}

Our patient population was predominantly African American ( $\mathrm{N}=126)$ with only two Hispanic and one Middle Eastern patient in our cohort. The median age at presentation was 68 years (IQR 63,75) with a median total Gleason sum of 8 (IQR 7.75,9). The median PSA level at presentation was $275 \mathrm{ng} / \mathrm{mL}$ (IQR 132,88). The median creatinine level and hematocrit were $1.2 \mathrm{mg} / \mathrm{dL}$ (IQR 1,1.6) and 37.9 (IQR 32.2,41.4), respectively (Table-1). The median follow-up was 26.6 months. The patients in this cohort presented over the course of 10 years (1999-2009), however our median follow-up time for these individuals was only 26.6 months.

Thirty-two patients presented with hydronephrosis with a median creatinine of $1.79 \mathrm{ng} / \mathrm{dL}$ (IQR 1.04, 4.12), two of whom required emergent dialysis. Thirteen of the 32 patients (40.6\%) who presented with hydronephrosis required intervention with either a percutaneous nephrostomy tube or ureteral stent placement. Intervention 
Table 1 - Demographics and presenting laboratory values (median).

\begin{tabular}{lc}
\hline Age & 68 \\
\hline PSA & $275 \mathrm{ng} / \mathrm{mL}$ \\
Race & \\
African American \% & 97.7 \\
Other \% & 2.3 \\
Gleason Sum & 8 \\
Creatinine & $1.2 \mathrm{mg} / \mathrm{dL}$ \\
Patients needing dialysis & 2 \\
Hematocrit \% & 37.9 \\
\hline
\end{tabular}

was required because of intractable flank pain or evidence of acute renal failure.

Screening CT scans were performed in 93 patients. Of those patients who underwent imaging at presentation 33/93 (35.5\%) were found to have lymphadenopathy suspicious for metastasis, $15 / 93(16.1 \%)$ had masses suspicious for visceral metastasis with the predominant site being the liver or lungs. Positive bone scans were identified in 118/127 (93\%) patients with 10/127 (7.9\%) exhibiting suspicion of cord compression (Table-2).

Table 2 - Findings on imaging within 30 days of diagnosis (\%).

\begin{tabular}{lc}
\hline Lymphadenopathy & 65.5 \\
Visceral metastases & 16.1 \\
Bone scan positive & 93 \\
Spinal Cord Compression & 7.9 \\
\hline
\end{tabular}

Six patients were lost to follow-up prior to obtaining the first post-treatment PSA level. Undetectable levels of PSA were achieved in 35/123 $(28.4 \%)$ patients. The median time for the first PSA rise above initial treatment PSA, if it occurred, was 6.8 months (IQR 3.1,11.2). The median PSA at time of biochemical failure was $18.69 \mathrm{ng} /$ mL (IQR 2.1, 186.2).

The 2-year and 5-year cancer specific survival for our patient cohort was $89.4 \%$ and $62.7 \%$, respectively (Figure-1). On univariate analysis creatinine at presentation $(p<0.05)$ and the presence of suspicious lymph nodes $(p=0.012)$ were independent predictors of PCa mortality. On multivariate analysis we were unable to identify any significant predictors of mortality. Using Kaplan-Meier survival analysis we compared the patients who presented with either lymphadenopathy or visceral metastasis to those who did not. The 5-year survival for patients with lymphadenopathy suspicious for metastatic disease on imaging compared to normal imaging was $42.5 \%$ and $75.4 \%$, respectively $(\mathrm{p}=0.074$ ) (Figure-2). When comparing those patients with imaging findings suspicious for visceral metastasis to those with normal imaging we found a 5-year survival of $27.5 \%$ and $65.3 \%$ respectively ( $p=$ 0.012) (Figure-3).

Unfortunately, we did not have access to death certificates or actual cause of death for the vast majority of the patients in this database.

\section{DISCUSSION}

The findings reported in the present study provide additional insight into the severe morbidity and mortality associated with $\mathrm{mPCa}$ in an underserved, predominantly African American population despite widespread use of PSA screening. In our cohort we identified 202 patients, 129

Figure 1 - Kaplan-Meier Cancer Specific Survival Curve.

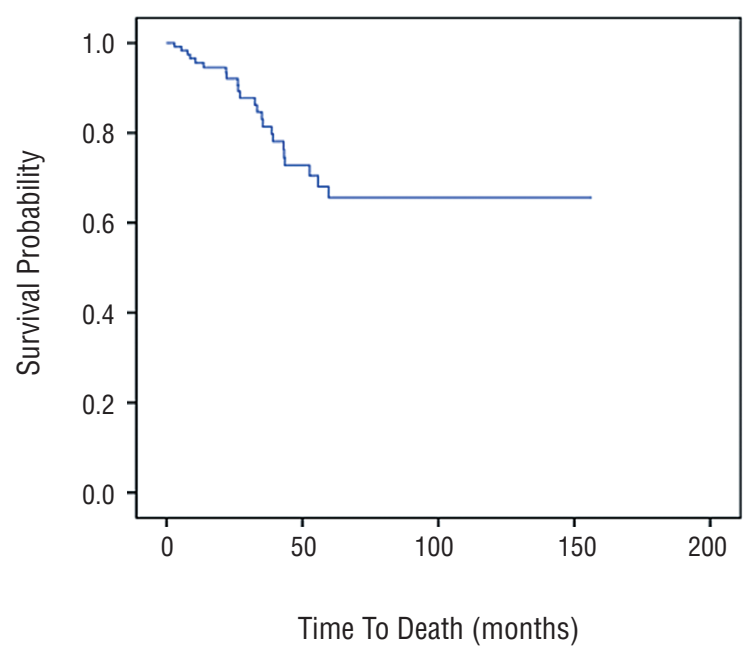


Figure 2 - Kaplan-Meier Survival Lymph Node Positive Disease Curve.

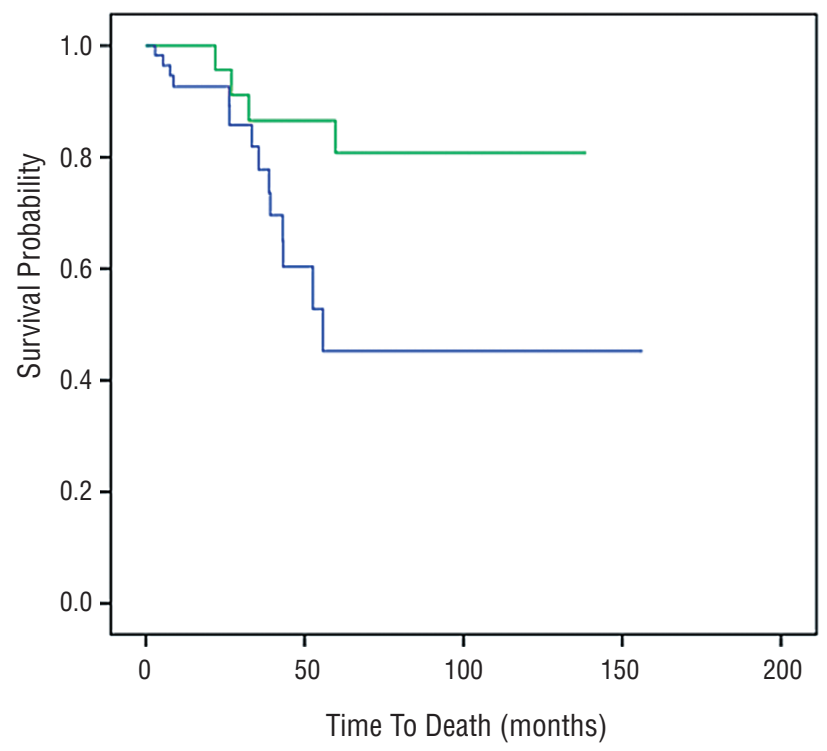

$\neg$ Lymph Node Negative $\neg$ Lymph Node Positive

evaluable based on our inclusion criteria, who were diagnosed with $\mathrm{mPCa}$ on initial presentation. The patients in this study were treated at an inner-city hospital that primarily serves individuals of lower SES often who have immigrated from third world countries in which PSA screening is largely unavailable. These patients presented with a significant cancer burden; 93\% of which had skeletal metastases identified on bone scan, 65.5\% had suspicious lymph nodes and $16.1 \%$ showing evidence of visceral metastasis on CT scan. Ten (7.9\%) of these patients had radiological evidence of spinal cord compression, which is consistent with currently reported numbers in patients with mPCa (7-10). As expected, we also demonstrated that patients presenting with evidence of visceral metastases had a significantly higher mortality rate. While one can see a clear separation in mortality rates between patients with and without lymphadenopathy at presentation, this was not statistically significant (p $=0.074$ ) likely due to the low number of patients in each group. In addition, imaging was only evaluated at time of presentation in these patients. Therefore, if our review was expanded we would have
Figure 3 - Kaplan-Meier Survival Visceral Metastasis Curve.

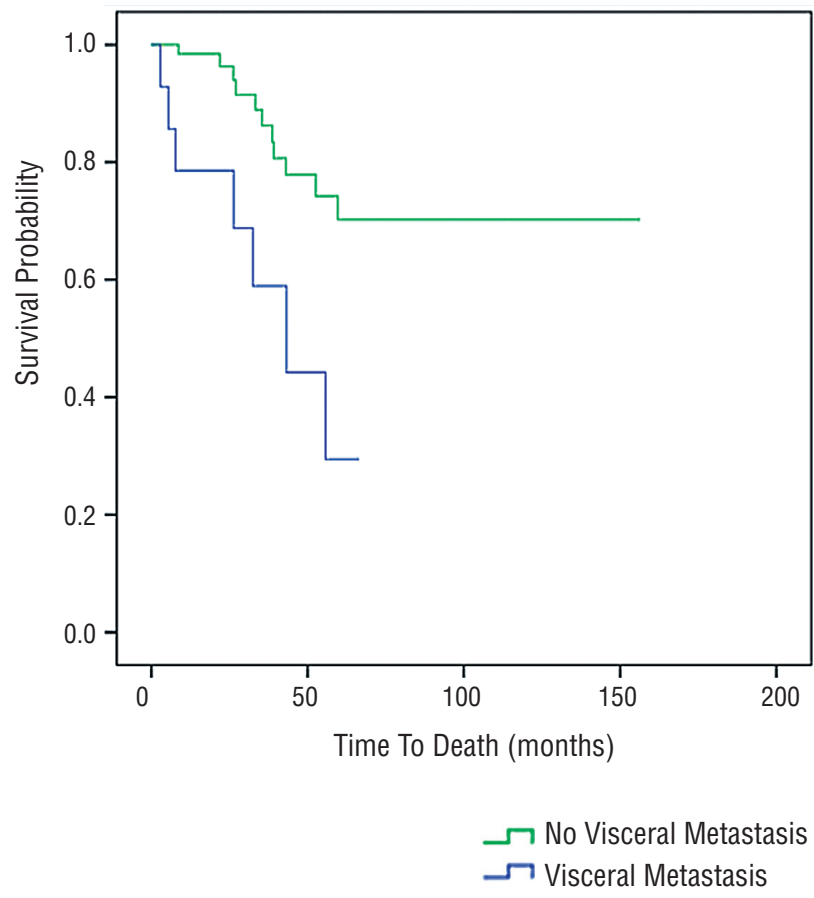

found higher rates of lymphadenopathy on later imaging, thus increasing the power of the analysis.

In previously reported studies with a more heterogeneous subset of patients, overall survival in men with metastatic disease was found to range from 5 to 7 years (11-14). Only $62.7 \%$ of our patient population was alive at 5 years. In spite of hormonal therapy, we demonstrated a median time to PSA rise above nadir of 6.8 months in this cohort. Ultimately, our findings reaffirm the notion that despite current therapies, those with distant metastases involving lymph nodes and/or visceral organs at the time of presentation have considerably poorer prognoses underscoring the importance of PSA screening in our male population.

Our patients in this study were predominately of African American descent, residing in an underserved area. In a SEER database study, Cheng et al. compared mortality rates from PCa based on SES and race. They demonstrated that higher SES is associated with a higher incidence of PCa but a lower mortality rate. Upon further analysis of patients with low SES they reported a higher mortality rate and when sub-stratified by 
race they discovered that mortality rates from this disease were substantially higher among African-American men when compared to other racial/ethnic groups (15). The conclusions of this study in conjunction with our findings reinforce the potential danger of eliminating PSA screening in the general population but particularly in high-risk groups.

Despite the fact that overall rates of $\mathrm{mPCa}$ appear to be declining, our results and the conclusions of other recent studies reaffirm the notion that at risk populations would suffer greatly from the elimination of PCa screening $(3,4,15-17)$. One such retrospective review, performed by Miller et al., examined 570 low-income uninsured men with the diagnosis of PCa. They demonstrated that $51 \%$ of these patients had a PSA greater than $10 \mathrm{ng} / \mathrm{mL}, 50 \%$ had a Gleason score of 7 or greater, 43\% had T2 or greater clinical stage, and $19 \%$ had metastatic disease at the time of diagnosis. They also showed that organ confined, low risk PCa did not increase over the 5-year period among these men (4). In our study we report a median PSA of $275 \mathrm{ng} / \mathrm{mL}$ and a median Gleason sum of 8 from an underserved population of men who presented with mPCa. Clearly, PCa remains a significant public health concern, in particular in African-American men living in underserved areas. Therefore, we strongly feel that in order to diminish the racial and socioeconomic inequalities with regards to PCa morbidity and mortality, this high-risk population may benefit from a more comprehensive screening effort on behalf of primary care physicians and urologists alike.

There are several limitations of the present study. Despite being a prospectively maintained database, this was a strictly observational, retrospective analysis demonstrating the experience at a single institution. In addition, this cohort was homogeneous in its racial/ethnic breakdown therefore generalizations regarding the specific features of mPCa may not be extrapolated to the likes of the general population from this data alone.

\section{CONCLUSIONS}

We describe a cohort of men from an underserved area with $\mathrm{mPCa}$ on initial presentation in the PSA screening era. Our results underscore the notion that despite widespread use of PSA screening, aggressive, life-threatening PCa still exists. Additional studies should be performed focusing more specifically on the effect of PSA screening in an underserved population.

\section{ABBREVIATIONS}

$\mathrm{PCa}=$ prostate cancer

$\mathrm{mPCa}=$ metastatic prostate cancer

PSA $=$ prostate specific antigen

SES $=$ socioeconomic status

$\mathrm{CT}=$ computed tomography

LHRH = luteinizing hormone releasing hormone

IQR = interquartile range

SEER = surveillance, epidemiology and end-results USPSTF $=$ U.S. preventative services task force PLCO $=$ prostate, lung, colorectal and ovarian

\section{CONFLICT OF INTEREST}

None declared.

\section{REFERENCES}

1. Howlander N, Noone AM, Krapcho M.: SEER Cancer Statistics Review. 1975-2008. Bethesda, MD: National Cancer Institute; 2011.

2. Shao YH, Demissie K, Shih W, Mehta AR, Stein MN, Roberts $\mathrm{CB}$, et al.: Contemporary risk profile of prostate cancer in the United States. J Natl Cancer Inst. 2009; 101: 1280-3.

3. Etzioni R, Gulati R, Falcon S, Penson DF: Impact of PSA screening on the incidence of advanced stage prostate cancer in the United States: a surveillance modeling approach. Med Decis Making. 2008; 28: 323-31.

4. Miller DC, Litwin MS, Bergman J, Stepanian S, Connor SE, Kwan L, et al.: Prostate cancer severity among low income, uninsured men. J Urol. 2009; 181: 579-83; discussion 5834.

5. Faria EF, Carvalhal GF, Vieira RA, Silva TB, Mauad EC, TobiasMachado $\mathrm{M}$, et al.: Comparison of clinical and pathologic findings of prostate cancers detected through screening versus conventional referral in Brazil. Clin Genitourin Cancer. 2011; 9: 104-8.

6. Jemal A, Siegel R, Ward E, Murray T, Xu J, Thun MJ: Cancer statistics, 2007. CA Cancer J Clin. 2007; 57: 43-66.

7. Bubendorf L, Schöpfer A, Wagner U, Sauter G, Moch H, Willi $N$, et al.: Metastatic patterns of prostate cancer: an autopsy study of 1,589 patients. Hum Pathol. 2000; 31: 578-83. 
8. Markman M: Early recognition of spinal cord compression in cancer patients. Cleve Clin J Med. 1999; 66: 629-31.

9. Fervenza FC, Wolanskyj AP, Eklund HE, Richardson RL: Brain metastasis: an unusual complication from prostatic adenocarcinoma. Mayo Clin Proc. 2000; 75: 79-82.

10. Benjamin R: Neurologic complications of prostate cancer. Am Fam Physician. 2002; 65: 1834-40.

11. Pound CR, Partin AW, Eisenberger MA, Chan DW, Pearson JD, Walsh PC: Natural history of progression after PSA elevation following radical prostatectomy. JAMA. 1999; 281: 1591-7.

12. Makarov DV, Humphreys EB, Mangold LA, Carducci MA, Partin AW, Eisenberger MA, et al.: The natural history of men treated with deferred androgen deprivation therapy in whom metastatic prostate cancer developed following radical prostatectomy. J Urol. 2008; 179: 156-61; discussion 161-2.

13. Yossepowitch 0, Bianco FJ Jr, Eggener SE, Eastham JA, Scher HI, Scardino PT: The natural history of noncastrate metastatic prostate cancer after radical prostatectomy. Eur Urol. 2007; 51: 940-7; discussion 947-8.

14. Ryan CJ, Elkin EP, Cowan J, Carroll PR: Initial treatment patterns and outcome of contemporary prostate cancer patients with bone metastases at initial presentation: data from CaPSURE. Cancer. 2007; 110: 81-6.
15. Cheng I, Witte JS, McClure LA, Shema SJ, Cockburn MG, John EM, et al.: Socioeconomic status and prostate cancer incidence and mortality rates among the diverse population of California. Cancer Causes Control. 2009; 20: 1431-40.

16. Tewari AK, Gold HT, Demers RY, Johnson CC, Yadav R, Wagner EH, et al.: Effect of socioeconomic factors on longterm mortality in men with clinically localized prostate cancer. Urology. 2009; 73: 624-30. Erratum in: Urology. 2009; 74: 239.

17. Adler NE, Newman K: Socioeconomic disparities in health: pathways and policies. Health Aff (Millwood). 2002; 21: 60-76.
Correspondence address: Andrew Winer, MD

Department of Urology SUNY Downstate Medical Center 450 Clarkson Ave. Brooklyn, NY 11203

Fax: +1 718 270-3848

E-mail: andrew.winer@downstate.edu 